doi:10.4149/neo_2018_161217N648

\title{
An extensive study of the mechanism of prostate cancer metastasis
}

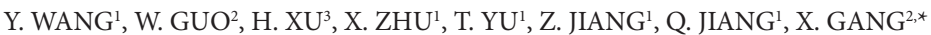

${ }^{1}$ Department of Orthopedics, The Second Hospital of Jilin University, Changchun, 130041, China; ${ }^{2}$ Department of Endocrinology and Metabolism, The First Hospital of Jilin University, Changchun, Jilin 130021, China; ${ }^{3}$ Department of Ophthalmology, The Second Hospital of Jilin University, Changchun, 130041, China

*Correspondence: gangxiaok2016@hotmail.com

Received December 17, 2016 / Accepted August 16, 2017

\begin{abstract}
The study aimed to identify the pivotal genes and pathways involved in prostate cancer metastasis. Using the expression profile dataset GSE7930, downloaded from the Gene Expression Omnibus (GEO) database, differentially expressed genes (DEGs) between primary and highly metastatic prostate cell samples were screened, followed by functional analysis and tumor associated genes (TAG) screening. Protein-protein interaction (PPI) network of DEGs was constructed and module analysis was performed. The expression of DEGs and pathway related genes were evaluated by PCR analysis and the migration ability of prostate tumor cells was observed after FABP4-siRNA blocking. Upregulated FABP4 and GK were significantly enriched in the PPAR signaling pathway, whereas downregulated IGFBP3 and THBS1 were involved in p53 signaling pathway. Among the identified DEGs, 4 downregulated genes (IGFBP3, NPP4B, THBS1, and PCDH1) and 2 upregulated genes (GJA1 and TUSC3) were TAGs. The module was associated with focal adhesion, ECM-receptor interaction, p53 signaling, and gap junction pathways with the hub node GJA1. After FABP4 silencing by siRNAs in LNcap and metastatic DU-145 cells, the numbers of migrated cells were all significantly declined. The expressions of IGFBP3, TP53 and PPAR were significantly lower in DU-145 cells than in LNcap cells. In conclusion, FABP4, IGFBP3, THBS1, and GJA1 were determined to be potential markers of prostate cancer cell metastasis, and P53, PPAR and gap junction pathways were found to play important roles in prostate cancer cell metastasis. This study may provide helpful guidelines for clinical management.
\end{abstract}

Key words: metastasis, prostate cancer, microarray profile, differentially expressed genes, tumor associated genes

Prostate cancer is the second most common malignancy with 250,000 new deaths every year $[1,2]$. It may show no apparent symptoms or mere urinary system disorders such as blood in the urine and difficulty in urinating. Moreover, serious symptoms such as bone pain in the vertebrae, spinal cord, pelvis, and ribs may occur due to metastasis of advanced prostate cancer to other organs of the body, especially the bones and lymph nodes, as well as invasion into local surrounding tissues including the rectum, bladder, and lower ureters $[3,4]$. Determination of the stage of prostate cancer, a reflection of the extent of cancer metastasis, is essential for evaluating the prognosis of patients with prostate cancer. Although there are numerous approaches such as prostate cancer screening, prostate-specific antigen (PSA) blood testing, and digital rectal exams for detecting and preventing prostate cancer, the impact of those technologies on reducing incidence and morbidity of prostate cancer has proven controversial and may even result in unnecessary harm to patients [5]. Therefore, the major priority now is to identify promising biomarkers specific for predicting the probability of prostate cancer metastasis.

In recent years, some molecular pathways and genes have been reported to elucidate the molecular mechanism of prostate cancer metastasis. Some pathways play important role in the metastatic process of prostate cancer; for instance, Hedgehog $(\mathrm{Hh})$ signaling has been reported to modulate prostate cancer metastasis [6]. Russell et al. reported that Stromal Cell-derived Factor-1/C-X-C Motif Chemokine Receptor 4 (SDF-1/CXCR4) pathway facilitates the metastasis of prostate cancer to bone [7]. Mitogenactivated protein kinase (MAPK) signaling pathway has been identified in metastatic prostate cancer samples [8], which promotes progression of the prostate cancer to advanced prostate cancer [9]. Besides, some genes have been found to play important role in prostate cancer metastasis. Secreted phosphoprotein 1 (SPPI) is reportedly involved in prostate 
cancer bone metastasis [3]. Developmentally regulated GTP-binding protein 1 (DRG1) [10], Raf kinase inhibitor protein (RKIP) [11], mitogen-activated protein kinase kinase 4/stress-activated protein/Erk kinase 1 (MKK4/SEK1) [12] have been demonstrated to suppress prostate cancer metastasis. Despite these studies, the extensive molecular mechanisms underlying metastatic prostate cancer development are still poorly understood.

In an attempt to reveal the genes and pathways that specifically play key roles in prostate cancer metastasis, differentially expressed genes (DEGs) were identified between primary and highly metastatic prostate cancer samples. The specific biological functions of the identified DEGs were subsequently studied by Gene ontology (GO) and Kyoto Encyclopedia of Genes and Genomes (KEGG) pathway enrichment analyses, accompanied by screening the tumor associated genes (TAG) and TF (Transcription Factor) genes from the DEGs. Furthermore, a protein-protein interaction (PPI) network was established to evaluate interactions among the proteins encoded by DEGs, followed by extracting modules from the network to unearth the pivotal proteins showing multiple interactions with others.

\section{Materials and methods}

Microarray data and preprocessing. The expression profile dataset GSE7930 deposited by Wong et al. [13] and based on the Affymetrix Human Genome U133A Array platform, was downloaded from the Gene Expression Omnibus (GEO) database (https://www.ncbi.nlm.nih.gov/ geo). Three primary prostate cancer subcutaneous tumor samples derived from the PC3-\#78 cell line (GSM155023, GSM155024, GSM155025) and three highly metastatic prostate subcutaneous tumor tissues derived from the PC-\#82 cell line (GSM155026, GSM155027, GSM155028) were used for expression profiling analysis. These prostate cancer cell lines were derived by the re-implantation of pMicro- 1 cells into mice, which were obtained by implanting the human cell line PC-3 into mice.

Gene probes were assigned to corresponding gene names according to the Affymetrix genechip probe annotation profile provided by Brain Array Lab, and the expression value was averaged when numerous probes corresponded to the same gene. Gene expression data were sequentially subjected to preprocessing procedures including background correction, quantile normalization, and probe summarization using the robust multiarray analysis (RMA) of affy package in R language [14].

Differentially expressed gene (DEG) screening. After data preprocessing, DEGs were identified between primary prostate cancer cell lines and highly metastatic prostate cancer cell lines using the empirical Bayes method of Linear Models for Microarray Analysis (LIMMA) package in $\mathrm{R}$ language. A false discovery rate $(\mathrm{FDR})<0.05$ and $|\log 2 \mathrm{FC}| \geq 1$ were regarded as the cut-off criteria.
GO and KEGG enrichment analysis for DEGs. For the purpose of providing an insight into exact biological functions related to the obtained DEGs, GO enrichment analysis was performed in terms of Biological Process (BP), Molecular Function (MF), and Cellular Component (CC) based on Gene Ontology database [15], with $\mathrm{p}<0.05$ as the strict cutoff. Besides, KEGG enrichment analysis based on KEGG database [16] was conducted to determine the signaling pathways with $\mathrm{p}<0.05$, which were enriched by the acquired DEGs.

Gene function annotation. The transcription factor database (TRANSFAC) is publicly available and is rich in information about TFs, TF binding sites, and DNA-binding profiles [17]. Based on the TFs information from TRANSFAC, the DEGs encoding TFs were picked out from the identified DEGs. TAGs including oncogenes and tumor suppressor genes (TSG) were also extracted from DEGs based on the TAG database [18] and TSGene database [19].

Protein-Protein interaction (PPI) network construction and module extraction. The Search Tool for the Retrieval of Interacting Genes (STRING) [20] is a database containing all the known and predicted protein interactions and associations. By mapping DEGs in the STRING database, the PPI network was built and visualized using Cytoscape software [21], in which the combined score of pairwise interaction was $\geq 0.9$. In the PPI network, the undirected link represented a pairwise interaction between proteins, which served as "nodes". "Degree" was defined as the number of interactions between a given protein and other proteins, and the protein with the highest degree was considered as a hub "node".

Subsequently, analysis of the constructed PPI network was performed to mine the module with a FDR $<0.05$ using the BioNet analytical tool that combines the data on transcriptome and functions with biological networks for an integrative network analysis, followed by KEGG enrichment analysis for module genes.

Quantitative reverse transcription-PCR (RT-PCR) analysis. The primary prostate tumor LNcap cells and metastatic prostate cancer DU-145 cells were respectively maintained in complete DMEM and MEM media (Gibco Laboratories, Grand Island, NY) at standard conditions. After culture, cells were collected by centrifugation at 1,000 rpm for $5 \mathrm{~min}$. The total RNA of cells was isolated by Trizol kit (Invitrogen, Carlsbad, CA) following the manufacturer's instructions.

Total $0.5 \mu \mathrm{g}$ RNA was reversely transcribed to cDNA by using TaKaRa cDNA Synthesis Kit (TAKARA, Japan). The expressions of IGFBP3, TP53 and PPAR were analyzed by PCR analysis. The PCR reaction volume was $20 \mu \mathrm{l}, 10 \mu \mathrm{l}$ SYBR Premix EX Taq (2x), $1 \mu \mathrm{l}$ forward primer, $1 \mu \mathrm{l}$ reverse primer, and $8 \mu \mathrm{l} \mathrm{cDNA}$. The primer sequences of IGFBP3, THBS1, GJA1, TP53 and PPAR are listed in Supplemental Table 1. PCR reaction was performed in Applied Biosystems ViiA7 PCR System as follows: $50^{\circ} \mathrm{C}$ for $3 \mathrm{~min}$ followed by 40 cycles of $95^{\circ} \mathrm{C}$ for $3 \mathrm{~min}, 95^{\circ} \mathrm{C}$ for $10 \mathrm{~s}, 60^{\circ} \mathrm{C}$ for $30 \mathrm{~s}$. The same experiments were performed in triplicate. 
FABP4 silencing by siRNA. LNcap and DU-145 cells were seeded in 12-well plates. Cells were cultured in antibiotic-free media until the density was up to $30-50 \%$. Then, cells were incubated with $5 \mu \mathrm{l}$ FABP 4 siRNA $(20 \mu \mathrm{M})$ in a final volume of $100 \mu$ diluted by serum-free media, followed by transfection with lipofectamine 2000. FABP4-siRNA (sense-siFABP4: 5'-GGAUGUGAUCACCAUUAAA-3'; antisense-siFABP4 5'-UUUAAUGGUGAUCACAUCC-3') and non-target control siRNA (sense-siNC: 5'-UUCUCCGAACGUGUCACGUTT-3', antisense-siNC: 5'-ACGUGACACGUUCGGAGAATT-3') were synthesized by Shanghai Tuoran biotechnology co. LTD, Shanghai, China. The expression of FABP4 in LNcap and DU-145 cells was observed after cells were cultured for $24 \mathrm{~h}$ and $48 \mathrm{~h}$, respectively.

Cell migration assay. After FABP4 silencing, the cell migration ability was assessed by using a 24-well transwell plate according to the manufacturer's instructions (Corning, NY, USA). After FABP4 siRNA transfection, total $3 \times 10^{4}$ cell/ well were seeded into the upper chamber in serum-free media. The lower chamber was filled with $700 \mu \mathrm{L}$ media supplemented with $10 \%$ FBS. After cells were incubated for $24 \mathrm{~h}$, cells that migrated through the surface of membrane were fixed with methanol and stained with hematoxylin for 15 min. Cells were counted by using CKX31 microscope (Olymp $\mu$ s, Lake Success, NY) at $\times 100$ magnification from three random fields.

Statistical analysis. All the data were expressed as mean \pm SEM (standard error of the mean) and analyzed by SPSS 22.0 software. Differences between groups were analyzed by $t$ test. $\mathrm{p}<0.05$ was considered as significant.

\section{Results}

DEGs analysis. We found 19 upregulated DEGs and 22 downregulated DEGs between the primary and highly metastatic prostate cell samples (Table 1).

Enrichment analyses for DEGs. The KEGG pathways enriched by the upregulated and downregulated DEGs are displayed in Table 2, showing that upregulated fatty acid binding protein 4 (FABP4) and glucokinase $(G K)$ were significantly enriched in peroxisome proliferator-activated receptor (PPAR) signaling pathway (Supplemental Figure 1), whereas downregulated insulin-like growth factor-binding protein 3 (IGFBP3) and thrombospondin 1 (THBS1) were significantly enriched in p53 signaling pathway (Supplemental Figure 2). As shown in Table 3, downregulated DEGs were closely related to several biological processes, including magnesium ion response, apoptotic cell clearance, regulation of macrophage activation, peptide cross-linking, negative regulation of endopeptidase activity, and cell adhesion, whereas upregulated DEGs were significantly linked to the regulation of behavior, response to hormone stimulus, tube development, and cardiac muscle cell proliferation.

TFs and TAG analyses. A range of analyses were conducted to classify DEGs based on their functions in tumors. Based
Table 1. DEGs screening.

\begin{tabular}{|c|c|c|c|}
\hline Category & Gene name & FDR value & $|\log 2 \mathrm{FC}|$ value \\
\hline \multirow{19}{*}{$\begin{array}{l}\text { Upregulated } \\
\text { DEGs }\end{array}$} & CCDC6 & 0.023673 & 1.031336 \\
\hline & DNM3 & 0.000476 & 3.209143 \\
\hline & FABP4 & 0.007272 & 1.353411 \\
\hline & GJA1 & 0.027124 & 1.399577 \\
\hline & $G K$ & 0.002738 & 1.154329 \\
\hline & GOLM1 & 0.022781 & 1.306406 \\
\hline & GPRC5C & 0.006742 & 1.300801 \\
\hline & HAPLN1 & 0.000511 & 1.489024 \\
\hline & HSD17B2 & 0.002566 & 1.541932 \\
\hline & IVNS1ABP & 0.009312 & 1.064485 \\
\hline & LMO4 & 0.024245 & 1.074291 \\
\hline & $M D K$ & 0.024245 & 1.766798 \\
\hline & NAP1L3 & 0.018714 & 1.309721 \\
\hline & PLA2G7 & 0.044923 & 1.132075 \\
\hline & $R B P 4$ & 0.027222 & 1.018216 \\
\hline & SPARC & 0.000476 & 2.047274 \\
\hline & TENM4 & 0.027387 & 1.252775 \\
\hline & TUSC3 & 0.02776 & 1.060124 \\
\hline & UTS2 & 0.000112 & 3.694131 \\
\hline \multirow{22}{*}{$\begin{array}{l}\text { Downregulated } \\
\text { DEGs }\end{array}$} & AMIGO2 & 0.022781 & 1.01386 \\
\hline & ANO1 & 0.007021 & 1.23848 \\
\hline & $A T P 1 B 1$ & 0.022781 & 1.2993 \\
\hline & CD70 & 0.011196 & 1.01488 \\
\hline & CEACAM5 & 0.006742 & 2.29386 \\
\hline & CEACAM6 & 0.018714 & 1.71073 \\
\hline & GPR87 & 0.000699 & 1.51978 \\
\hline & $I G F B P 3$ & 0.024245 & 1.11962 \\
\hline & IGFBP6 & 0.024245 & 1.10129 \\
\hline & $I N P P 4 B$ & 0.029642 & 1.26662 \\
\hline & KRT7 & 0.005608 & 1.14847 \\
\hline & MT1M & 0.009713 & 1.12131 \\
\hline & PCDH1 & 0.026083 & 1.33697 \\
\hline & $P I 3$ & 0.009312 & 1.49302 \\
\hline & $R B P M S$ & 0.000511 & 1.27176 \\
\hline & SCG5 & 0.019963 & 1.47413 \\
\hline & SLPI & 0.000668 & 2.03183 \\
\hline & TFF2 & 0.024245 & 1.18254 \\
\hline & TGM2 & 0.022832 & 1.76294 \\
\hline & THBS1 & 0.002968 & 1.7228 \\
\hline & TNFAIP6 & 0.023673 & 1.10826 \\
\hline & TNFRSF $11 B$ & 0.00199 & 2.32087 \\
\hline
\end{tabular}

DEGs, differentially expressed genes

Table 2. KEGG pathway enrichment analysis.

\begin{tabular}{llccl}
\hline $\begin{array}{l}\text { Regula- } \\
\text { tion }\end{array}$ & KEGG Pathway & $\begin{array}{c}\text { Gene } \\
\text { Counts }\end{array}$ & p-value & Gene List \\
\hline Up & PPAR signaling pathway & 2 & 0.003747 & FABP4,GK \\
Down & p53 signaling pathway & 2 & 0.002675 & IGFBP3,THBS1 \\
\hline
\end{tabular}

KEGG, Kyoto Encyclopedia of Genes and Genomes

on the DEGs, downregulated genes like IGFBP3, natriuretic peptide $4 \mathrm{~B}$ (NPP4B), THBS1, and protocadherin-1 (PCDH1), and upregulated genes like Gap junction alpha-1 protein 
Table 3. GO enrichment analysis.

\begin{tabular}{|c|c|c|c|c|c|}
\hline Category & GO ID & Term & $\begin{array}{l}\text { Gene } \\
\text { Counts }\end{array}$ & p-value & Gene List \\
\hline \multirow[t]{9}{*}{ Down } & GO:0032026 & response to magnesium ion & 2 & 0.0000682156 & THBS1, \\
\hline & & & & & TNFRSF11B \\
\hline & GO:0043277 & apoptotic cell clearance & 2 & 0.000393871 & TGM2,THBS1 \\
\hline & GO:0043030 & regulation of macrophage activation & 2 & 0.000473712 & TFF2,THBS1 \\
\hline & GO:0018149 & peptide cross-linking & 2 & 0.000704749 & TGM2,THBS1 \\
\hline & GO:0010951 & negative regulation of endopeptidase activity & 3 & 0.000743516 & PI3,SLPI,THBS1 \\
\hline & GO:0046850 & regulation of bone remodeling & 2 & 0.001040601 & INPP4B, TNFRSF11B \\
\hline & GO:0007155 & cell adhesion & 6 & 0.001805051 & AMIGO2, ATP1B1,PCDH1,TGM2, \\
\hline & & & & & THBS1,TNFAIP6 \\
\hline \multirow[t]{4}{*}{$\mathrm{Up}$} & GO:0050795 & regulation of behavior & 3 & 0.000773064 & $M D K, P L A 2 G 7, U T S 2$ \\
\hline & GO:0060038 & cardiac muscle cell proliferation & 2 & 0.001006527 & RBP4,TENM4 \\
\hline & GO:0009725 & response to hormone stimulus & 5 & 0.001207102 & GJA1,MDK,RBP4,SPARC,UTS2 \\
\hline & GO:0035295 & tube development & 4 & 0.002248938 & $\begin{array}{l}\text { GJA1, LMO4, } \\
\text { RBP4,SPARC }\end{array}$ \\
\hline
\end{tabular}

GO, gene ontology

Table 4. TAG and TF analysis.

\begin{tabular}{lccl}
\hline & TF counts & TAG counts & TAG genes \\
\hline Down & 0 & 4 & IGFBP3, NPP4B, THBS1, PCDH1 \\
Up & 0 & 2 & GJA1,TUSC3 \\
\hline
\end{tabular}

TF, Transcription Factor; TAG, Tumor Associated Genes

(GJA1) and tumor suppressor candidate 3 (TUSC3) were determined to be TAGs (Table 4), whereas no DEG associated TF was identified.

PPI network and module analysis. As shown in Figure 1, the PPI network was constructed with DEGs, the degree of nodes (proteins) in the PPI network was calculated, and the nodes with degrees $\geq 10$ were listed in descending order as follows: urotensin-2 (UTS2, degree $=47)$, secreted protein acidic and rich in cysteine (SPARC, degree $=32$ ), estradiol 17-beta-dehydrogenase $2(\mathrm{HSD} 17 \mathrm{~B} 2$, degree $=29)$, FABP4 $($ degree $=29)$, THBS1 $($ degree $=28)$, GJA1 $($ degree $=27)$, lipoprotein-associated phospholipase $A_{2}$ (PLA2G7, degree = 19), IGFBP3 (degree=17), inositol polyphosphate-4-phosphatase B (INPP4B, degree =15), and LIM domain only 4 (LMO4, degree $=11)$.

The module extracted from the PPI network included 28 nodes with the hub "GJA1" (degree $=7$, Figure 2 ), and subsequent KEGG analysis showed that the genes included in the module were significantly associated with focal adhesion, ECM-receptor interaction, p53 signaling pathway, melanoma, arrhythmogenic right ventricular cardiomyopathy (ARVC), and gap junction pathways (Table 5).

The expression of IGFBP3, TP53 and PPAR by RT-PCR analysis. The expression of IGFBP3, TP53 and PPAR genes was validated by RT-PCR analysis. Compared with the primary prostate cancer LNcap cells, the relative expression of IGFBP3 was significantly declined in metastatic DU-145 cells ( 1.00 vs. $0.72 ; \mathrm{p}<0.001)$. Similar findings were found in the expression of TP53 and PPAR genes. The expression of TP53 and PPAR was significantly lower in DU-145 cells than in LNcap cells $(\mathrm{p}<0.01, \mathrm{p}<0.001)$ (Figure 3).

The expression of FABP4 and cell migration after siRNA transfection. After transfected with FABP4-siRNA at $24 \mathrm{~h}$ and $48 \mathrm{~h}$, the expressions of FABP 4 were evaluated. As shown in Figure $4 \mathrm{~A}$ and $\mathrm{B}$, the expression of $F A B P 4$ was significantly declined in LNcap cells at $24 \mathrm{~h}$ and $48 \mathrm{~h}$ after FABP4-siRNA silencing $(\mathrm{p}<0.01)$. The inhibitive rate of FABP4 expression in DU-145 cells was more significant. Compared with cells transfected with non-target siRNA, DU-145 cells showed a significant decline of FABP4 expression after siRNA blocking $(\mathrm{p}<0.001$, Figure 4C, 4D). Besides, after FABP4-siRNA transfection, the number of migrated cells was significantly declined in LNcap and DU-145 cells at $24 \mathrm{~h}$ and $48 \mathrm{~h}$ (all $\mathrm{p}<0.001$ ) (Figure $4 \mathrm{E}-\mathrm{H}$ ).

\section{Discussion}

Prostate cancer remains a threat to peoples' health. Far worse is its moderate metastatic propensity to spread to other organs of the body. We aimed to unravel the molecular mechanism underlying prostate cancer metastasis. In a screen for DEGs implicated in metastasis, 19 upregulated and 22 downregulated DEGs were acquired between the primary and highly metastatic prostate cancer samples. A range of bioinformatics approaches was utilized to analyze the exact function of DEGs in order to identify potential biomarkers of prostate cancer metastasis.

FABP4, also termed aP2 (adipocyte protein 2), is a carrier protein mainly residing in adipocytes and macrophages. Emerging studies have demonstrated that targeting this protein might be conducive to treat various diseases such as asthma, atherosclerosis, and diabetes [22-24]. A recent 


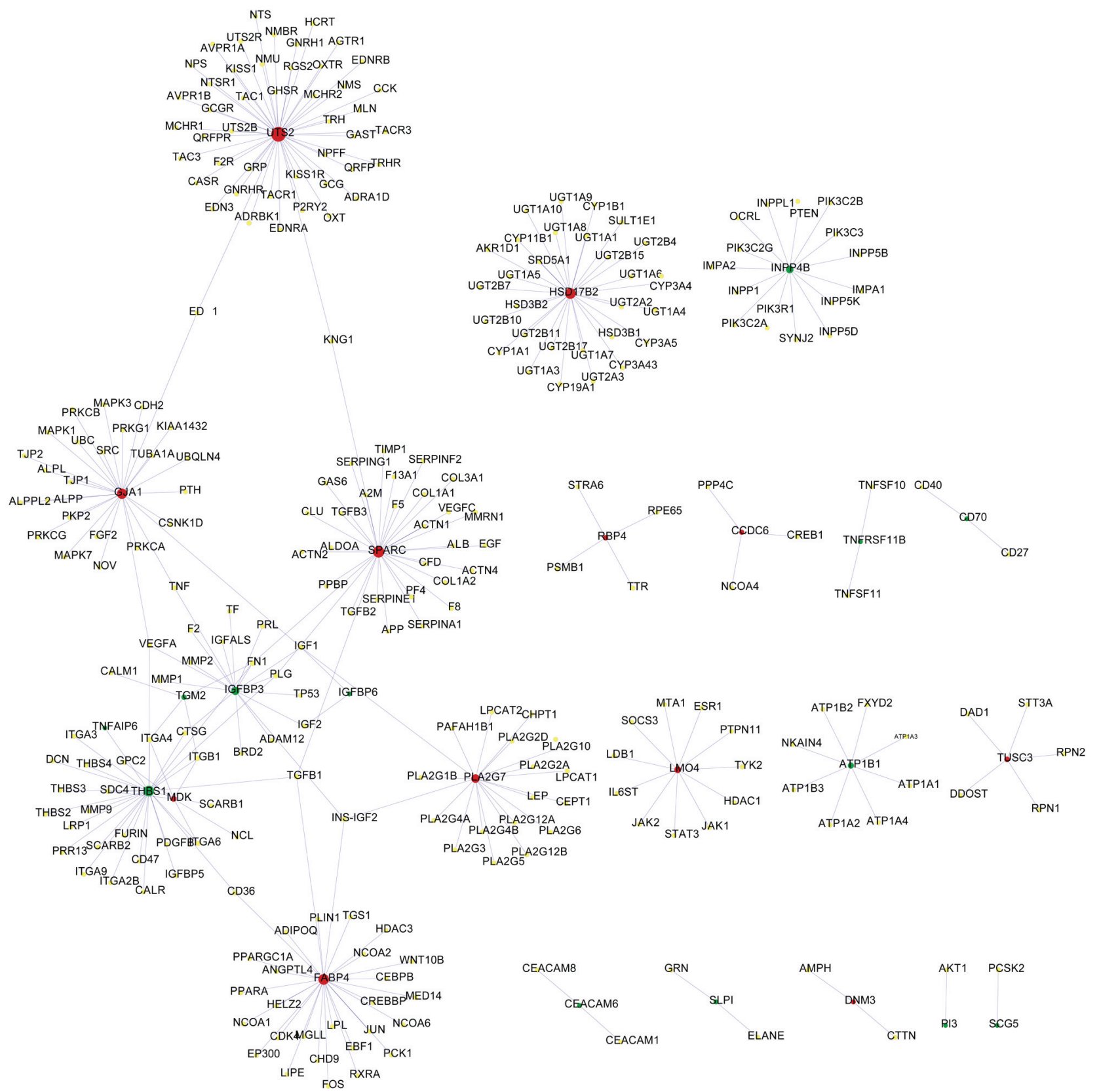

Figure 1. Protein-Protein interaction network analysis. Red node represents upregulated DEGs; green node represents downregulated DEGs; yellow node represents non-DEGs. DEGs, differentially expressed genes

study showed that FABP4 was upregulated in bone metastasis samples from prostate cancer patients and that FABP4 pathway might play an important role in the aggression of bone metastasis [25]. Similarly, the present study showed that FABP4 expression was significantly increased in highly metastatic cells compared with that in the primary cell line, and was enriched in PPAR pathway, suggesting that FABP4 might promote prostate cancer metastasis through the PPAR pathway. In order to validate the findings based on micro- array data analysis, we performed the experimental validation. Our data showed that the migration ability of prostate tumor cells was significantly declined after FABP4 silencing, which indicates the significant role of FABP4 in mediating prostate cancer cell migration. However, in the present study, the expression of PPAR was significantly declined in DU-145 and LNcap cells. Whether FABP4 plays a key role in the cell migration through PPAR pathway should be further analyzed. 
Table 5. KEGG enrichment analysis for the module.

\begin{tabular}{lccll}
\hline KEGG Pathway & $\begin{array}{c}\text { Gene } \\
\text { Counts }\end{array}$ & p-value & Upregulated genes & Downregulated genes \\
\hline Focal adhesion & 6 & 0.0000191 & MAPK1, COL3A1 & FN1, THBS1, ITGA3 \\
ECM-receptor interaction & 4 & 0.000107 & COL3A1 & THBS1, ITGA3 \\
Bladder cancer & 3 & 0.000258 & MAPK1, MMP1 & THBS1 \\
Pathways in cancer & 6 & 0.000294 & MAPK1, MMP1 & FGF2, FN1, ITGA3 \\
p53 signaling pathway & 3 & 0.001071 & & IGFBP3, THBS1 \\
Melanoma & 3 & 0.001215 & MAPK1 & CDH2, ITGA3 \\
Arrhythmogenic right ventricular cardiomyopathy (ARVC) & 3 & 0.00137 & GJA1 & FGF2, FN1, ITGA3 \\
Gap junction & 3 & 0.002408 & TUBA1A, MAPK, GJA1 & \\
Regulation of actin cytoskeleton & 4 & 0.003454 & MAPK1 & \\
Aldosterone-regulated sodium reabsorption & 2 & 0.007112 & MAPK1 & \\
\hline
\end{tabular}

KEGG, Kyoto Encyclopedia of Genes and Genomes

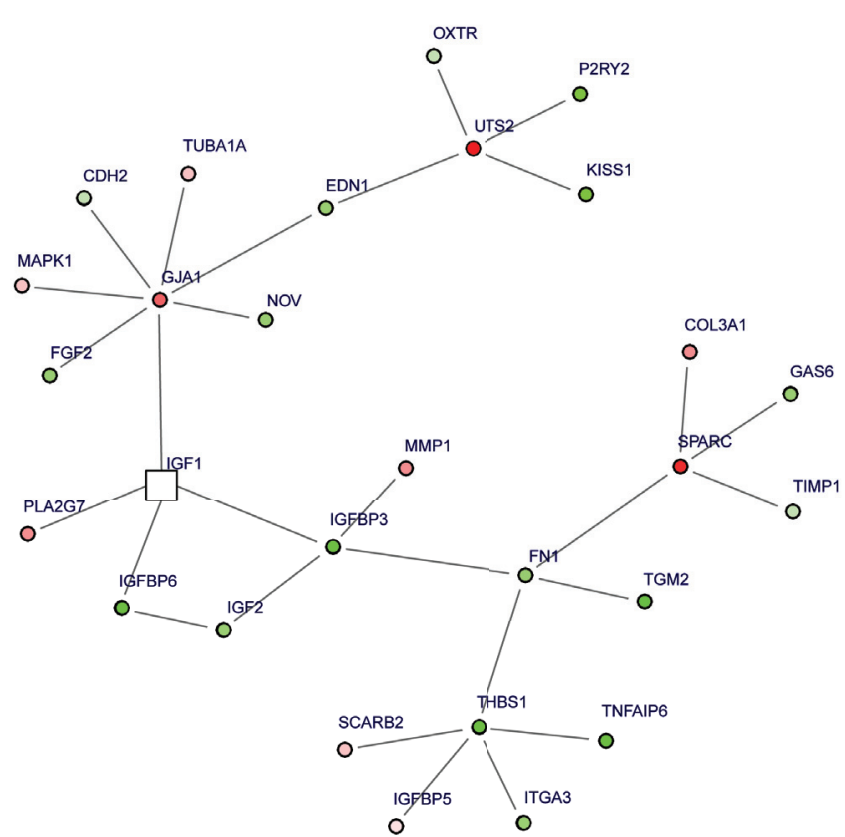

Figure 2. The module extracted from the Protein-Protein interaction network. The color depth of the node is positively associated with the fold change of the node gene. Red node, upregulated gene; green node, downregulated gene; white node, genes with significant changes in expression; square nodes, unimportant genes; round nodes, important genes.

IGFBP3 belongs to the IGFBP3 family with the characteristic IGFBP domain, and often functions in a complex with insulin-like growth factor [26]. Accumulating evidences based on findings from epidemiological studies and in vivo studies have revealed that IGFBP3 is a metastasis-suppressed gene in prostate cancer and could serve as a potential biomarker for its diagnosis and prognosis [27-29]. In the present study, IGFBP3 was found to be downregulated in highly metastatic cancer cells, which was determined by PCR analysis. PCR analysis showed that the expression of IGFBP3 was significantly lower in metastatic DU-145 cells than in the primary LNcap cells. Besides, IGFBP3 was identified as a
A
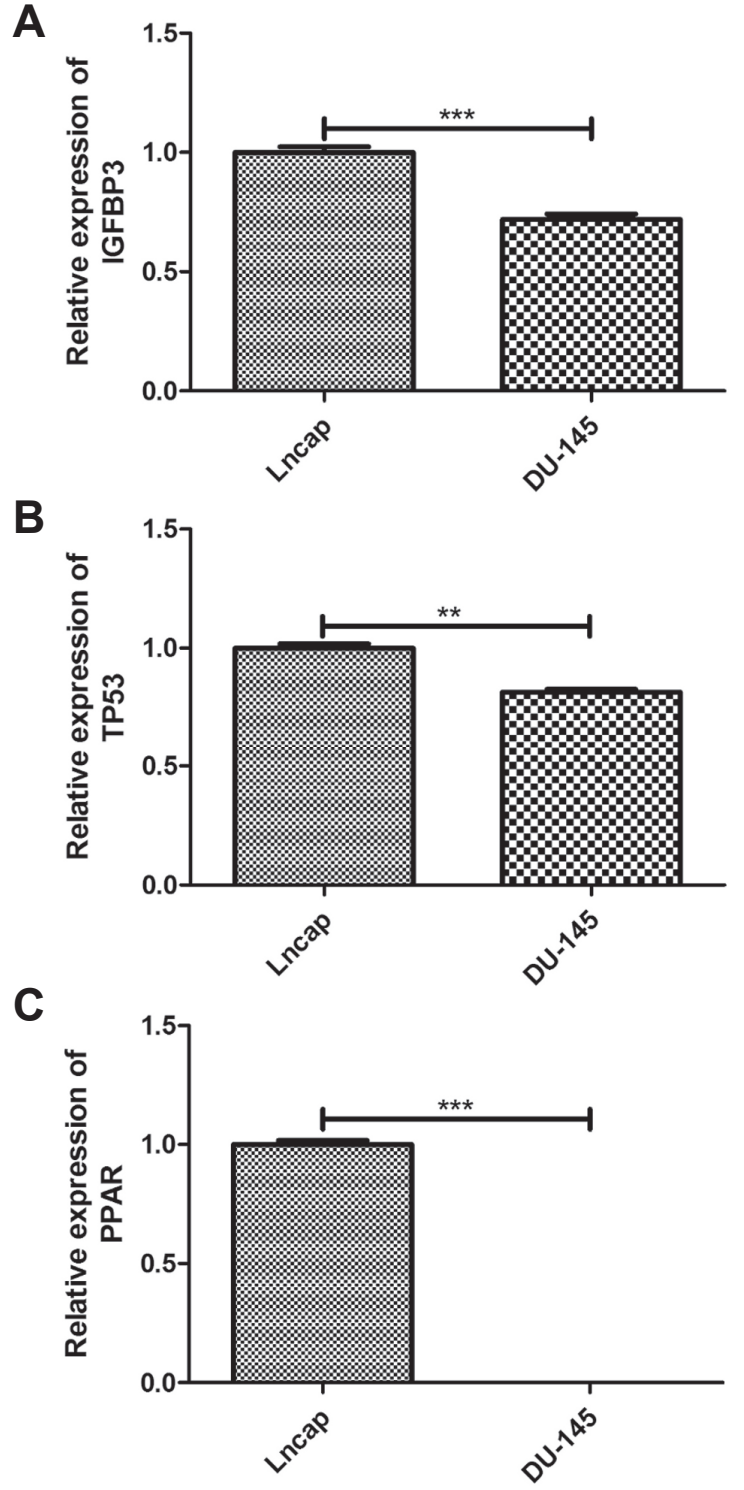

Figure 3. The expression of IGFBP3, TP53, and PPAR in primary LNcap and metastatic DU-145 cells; A: relative expression of IGFBP3; B: relative expression of TP53; C: relative expression of PPAR; ${ }^{* *} \mathrm{p}<0.01,{ }^{* *} \mathrm{p}<0.001$ 
TAG, which indicated the inhibitory role of IGFBP3 in prostate cancer metastasis. Besides, the downregulated genes, IGFBP3 and THBS1, were mainly enriched in the p53 pathway by bioinformatic analysis. The p53 signaling pathway has been considered a critical determinant of anti-tumor responses and expression of the p53 tumor suppressor gene is known to result in prostate cancer cell death through apoptotic and autophagic signaling pathways [30]. Inactivation of the p53 signaling pathway induced by mdm2 (murine double minute-2) overexpression could facilitate tumor progression in prostate cancer [31]. THBS1, encoding a matrix glycoprotein, is involved in interactions between cells, and between cells and the matrix. In consistence with the result of this study, THBS1 is known to inhibit tumor-related angiogenesis by regulating p53 [32] and repress tumor cell growth by activating transforming growth factor $\beta$ in tumor cells [33]. In this study, THBS1 was a critical hub protein in the PPI network and was enriched in apoptotic cell clearance, regulation of macrophages, and the p53 signaling pathway, indicating that the downregulated THBS1 might inhibit the apoptotic and autophagic signaling pathway to facilitate growth of metastatic prostate cancer cells. Besides, IGFBP 3 has been found to induce apoptosis in metastatic prostate cancer cells [34]. IGFBP3 was downregulated and also enriched in the p53 signaling pathway, indicating that the downregulated IGFBP3 might inactivate the p53 apoptotic signaling pathway by inhibiting apoptosis in metastatic prostate cancer cells. TP53 gene is a tumor suppressor that encodes $\mathrm{P} 53$ protein and is mutated in various human tumors [35]. In the present study, PCR analysis showed that the expression of TP53 gene is significantly decreased in the metastatic DU-145 cells compared with the LNcap cells, suggesting $\mathrm{p} 53$ signaling pathway was inhibited in metastatic DU-145 cells.
A

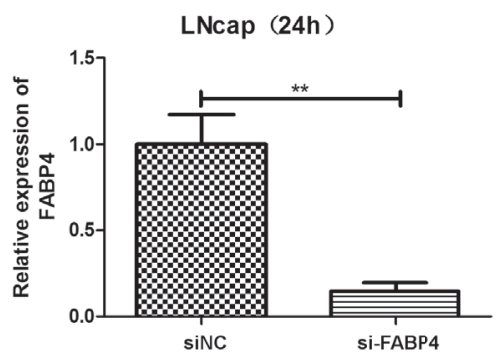

C

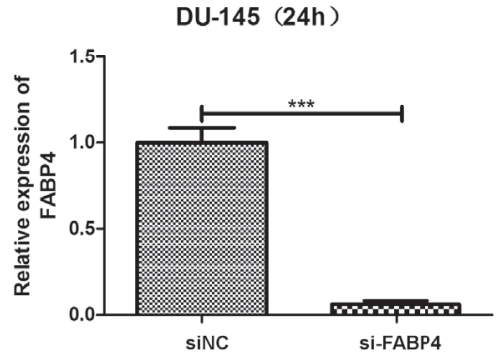

E

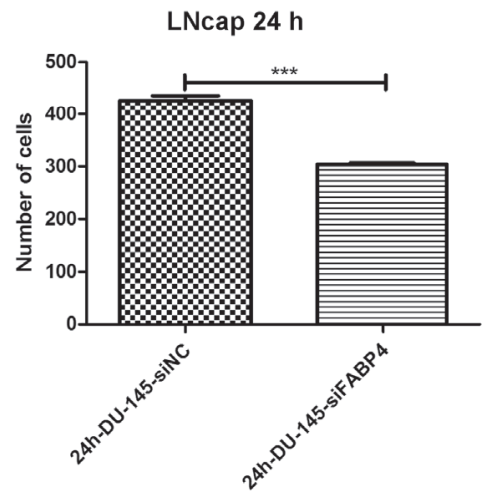

G

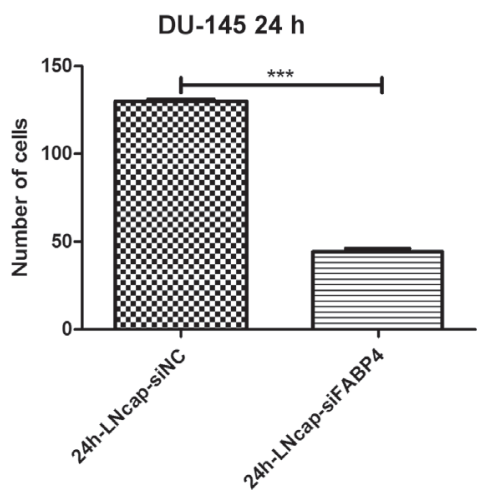

B

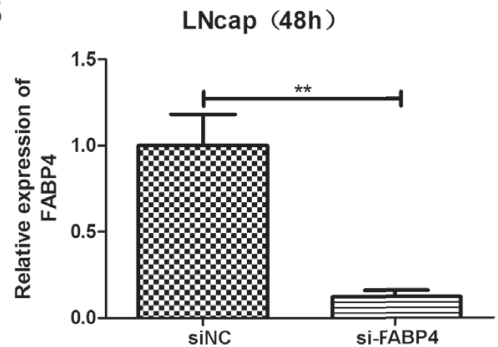

D

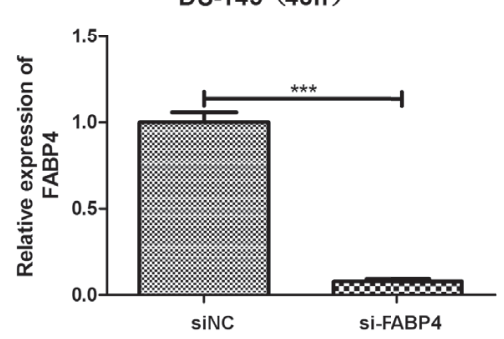

$\mathbf{F}$

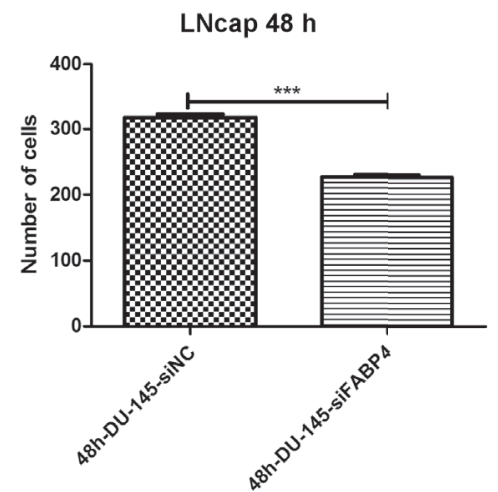

H

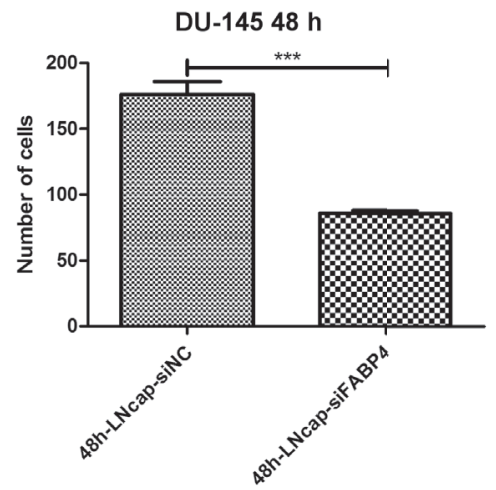

Figure 4. The expression of FABP4 and the migrated cells count after siRNA transfection A-D: the expression of FABP4 after siRNA transfection; E-H: the number of migrated cells after siRNA transfection; ${ }^{* *} \mathrm{p}<0.001$.

From the PPI network constructed in this study, a module was extracted and further analyzed by GO analysis which showed that the module was closely associated with focal adhesion, ECM-receptor interaction, p53 signaling pathway, melanoma, arrhythmogenic right ventricular cardiomyopathy, and gap junction pathways, 
indicating the critical roles of these pathways in prostate cancer metastasis. The hub gene of the module was upregulated GJA1, which was enriched in the gap junction pathway and identified as a TAG in the study. The protein encoded by GJA1 termed as connexin 43, is a major gap junction component, and plays a role in tumor invasion and progression [36]. GJAI is reportedly involved in early cancer invasion in prostate cancer $[37,38]$, indicating that GJAI might facilitate progression of prostate cancer cells by mediating the gap junction pathway and might be a promising biomarker for prostate cancer metastasis treatment.

Although we have made efforts to review the Gene Expression Omnibus (GEO) database and The Cancer Genome Atlas (TCGA) database, we have not found any data about metastatic prostate tumor samples. Moreover, we face difficulties in collecting metastatic prostate tumor samples in the clinic. Therefore, more experiments based on human metastatic prostate tumor samples are needed to verify our findings.

In conclusion, FABP4, IGFBP3, THBS1, and GJA1 might be potential biomarker candidates for diagnosing prostate cancer and potential targets for developing effective therapies to suppress cancer metastasis. P53, PPAR, and gap junction pathways could play an important role in prostate cancer invasion and metastasis. The findings from this study could contribute to a better understanding of the mechanism underlying prostate cancer metastasis and provide guidelines for its clinical management. Further experiments based on human metastatic prostate tumor samples are needed to verify our findings.

Supplementary information is available in the online version of the paper.

Acknowledgements: This work was supported by the Natural Science Foundation of China (81502190 to X. G.), Bethune B Plan of Jilin University (2015418 to X.G.) and The Sixth Young Foundation of The First Hospital of Jilin University (JDYY52015010 to X. G.), Youth Science and technology training program of Health and Family Planning Commission of Jilin Province (2016Q027 to X. G.), and Youth Science and technology training program of Health and Family Planning Commission of Jilin Province (16Q031 to Y. W.).

\section{References}

[1] JEMAL A, BRAY F, CENTER MM, FERLAY J, WARD E et al. Global cancer statistics. CA Cancer J Clin 2011; 61: 69-90. doi: 10.3322/caac. 20107

[2] HEIDENREICH A, BELLMUNT J, BOLLA M, JONIAU S, MASON M et al. EAU guidelines on prostate cancer. Part 1: screening, diagnosis, and treatment of clinically localised disease. Eur Urol 2011; 59: 61-71. doi: 10.1016/j.eururo.2010.10.039
[3] LOGOTHETIS CJ, LIN SH. Osteoblasts in prostate cancer metastasis to bone. Nat Rev Cancer 2005; 5: 21-28. doi: $10.1038 / \mathrm{nrc} 1528$

[4] VAN DER CRUIJSEN-KOETER IW, VIS AN, ROOBOL MJ, WILDHAGEN MF, DE KONING HJ et al. Comparison of screen detected and clinically diagnosed prostate cancer in the European randomized study of screening for prostate cancer, section Rotterdam. J Urol 2005; 174: 121-125. doi: 10.1097/01.ju.0000162061.40533.0f

[5] QASEEM A, BARRY MJ, DENBERG TD, OWENS DK, SHEKELLE P. Screening for prostate cancer: a guidance statement from the Clinical Guidelines Committee of the American College of Physicians. Ann Intern Med 2013; 158: 761-769. doi: 10.7326/0003-4819-158-10-201305210-00633

[6] KARHADKAR SS, BOVA GS, ABDALLAH N, DHARA S, GARDNER D et al. Hedgehog signalling in prostate regeneration, neoplasia and metastasis. Nature 2004; 431: 707-712. doi: $10.1038 /$ nature 02962

[7] TAICHMAN RS, COOPER C, KELLER ET, PIENTA KJ, TAICHMAN NS et al. Use of the Stromal Cell-derived Factor-1/ CXCR4 Pathway in Prostate Cancer Metastasis to Bone. Cancer Res 2002; 62: 1832-1837.

[8] CHANDRAN UR, MA C, DHIR R, BISCEGLIA M, LYONS-WEILER $\mathrm{M}$ et al. Gene expression profiles of prostate cancer reveal involvement of multiple molecular pathways in the metastatic process. BMC Cancer 2007; 7: 64. doi: 10.1186/1471-2407-7-64

[9] GIOELI D, MANDELL JW, PETRONI GR, FRIERSON HF, WEBER MJ. Activation of Mitogen-Activated Protein Kinase Associated with Prostate Cancer Progression. Cancer Res 1999; 59: 279.

[10] BANDYOPADHYAY S, PAI SK, GROSS SC, HIROTA S, HOSOBE S et al. The Drg-1 Gene Suppresses Tumor Metastasis in Prostate Cancer. Cancer Res 2003; 63: 1731-1736.

[11] FU Z, SMITH PC, ZHANG L, RUBIN MA, DUNN RL et al. Effects of raf kinase inhibitor protein expression on suppression of prostate cancer metastasis. J Natl Cancer Inst 2003; 95: 878-889.

[12] YOSHIDA BA, DUBAUSKAS Z, CHEKMAREVA MA, CHRISTIANO TR, STADLER WM et al. Mitogen-activated protein kinase kinase 4/stress-activated protein/Erk kinase 1 (MKK4/SEK1), a prostate cancer metastasis suppressor gene encoded by human chromosome 17. Cancer Res 1999; 59: 5483-5487.

[13] WONG SY, HAACK H, KISSIL JL, BARRY M, BRONSON RT et al. Protein $4.1 \mathrm{~B}$ suppresses prostate cancer progression and metastasis. Proc Natl Acad Sci U S A 2007; 104: 12784 12789. doi: 10.1073/pnas.0705499104

[14] IRIZARRY RA, HOBBS B, COLLIN F, BEAZER-BARCLAY YD, ANTONELLIS KJ et al. Exploration, normalization, and summaries of high density oligonucleotide array probe level data. Biostatistics 2003; 4: 249-264. doi: 10.1093/biostatistics/4.2.249

[15] ASHBURNER M, BALL CA, BLAKE JA, BOTSTEIN D, BUTLER $\mathrm{H}$ et al. Gene ontology: tool for the unification of biology. The Gene Ontology Consortium. Nat Genet 2000; 25: 25-29. doi: 10.1038/75556 
[16] KANEHISA M, GOTO S. KEGG: Kyoto encyclopedia of genes and genomes. Nucleic Acids Res 2000; 28: 27-30.

[17] WINGENDER E. The TRANSFAC project as an example of framework technology that supports the analysis of genomic regulation. Brief Bioinform 2008; 9: 326-332. doi: 10.1093/ bib/bbn016

[18] CHEN JS, HUNG WS, CHAN HH, TSAI SJ, SUN HS. In silico identification of oncogenic potential of fyn-related kinase in hepatocellular carcinoma. Bioinformatics 2013; 29: 420-427. doi: 10.1093/bioinformatics/bts715

[19] ZHAO M, SUN J, ZHAO Z. TSGene: a web resource for tumor suppressor genes. Nucleic Acids Res 2013; 41: 970-976. doi: 10.1093/nar/gks937

[20] FRANCESCHINI A, SZKLARCZYK D, FRANKILD S, KUHN M, SIMONOVIC M et al. STRING v9.1: protein-protein interaction networks, with increased coverage and integration. Nucleic Acids Res 2013; 41: D808-815. doi: 10.1093/ nar/gks1094

[21] SAITO R, SMOOT ME, ONO K, RUSCHEINSKI J, WANG PL et al. A travel guide to Cytoscape plugins. Nat Methods 2012; 9: 1069-1076. doi: 10.1038/nmeth.2212

[22] GHELFI E, YU C-W, ELMASRI H, TERWELP M, LEE CG et al. Fatty acid binding protein 4 regulates VEGF-induced airway angiogenesis and inflammation in a transgenic mouse model: implications for asthma. Am J Pathol 2013; 182: 1425-1433. doi: 10.1016/j.ajpath.2012.12.009

[23] PEETERS W, DE KLEIJN DP, VINK A, VAN DE WEG S, SCHONEVELD AH et al. Adipocyte fatty acid binding protein in atherosclerotic plaques is associated with local vulnerability and is predictive for the occurrence of adverse cardiovascular events. Eur Heart J 2011; 32: 1758-1768. doi: 10.1093/eurheartj/ehq387

[24] TORUNER F, ALTINOVA AE, AKTURK M, KAYA M, ARSLAN E et al. The relationship between adipocyte fatty acid binding protein-4, retinol binding protein-4 levels and early diabetic nephropathy in patients with type 2 diabetes. Diabetes Res Clin Pract 2011; 91: 203-207. doi: 10.1016/j.diabres.2010.11.011

[25] HERROON MK, RAJAGURUBANDARA E, HARDAWAY AL, POWELL K, TURCHICK A et al. Bone marrow adipocytes promote tumor growth in bone via FABP4-dependent mechanisms. Oncotarget 2013; 4: 2108-2123. doi: 10.18632/ oncotarget. 1482

[26] RENEHAN AG, ZWAHLEN M, MINDER C, O'DWYER ST, SHALET SM et al. Insulin-like growth factor (IGF)-I, IGF binding protein-3, and cancer risk: systematic review and meta-regression analysis. Lancet 2004; 363: 1346-1353. doi: 10.1016/S0140-6736(04)16044-3

[27] MEHTA HH, GAO Q, GALET C, PAHARKOVA V, WAN $\mathrm{J}$ et al. IGFBP-3 is a metastasis suppression gene in prostate cancer. Cancer Res 2011; 71: 5154-5163. doi: 10.1158/00085472.CAN-10-4513
[28] PRAGER AJ, PENG CR, LITA E, MCNALLY D, KAUSHAL A et al. Urinary aHGF, IGFBP3 and OPN as diagnostic and prognostic biomarkers for prostate cancer. Biomark Med 2013; 7: 831-841. doi: 10.2217/bmm.13.112

[29] SHARIAT SF, LAMB DJ, KATTAN MW, NGUYEN C, KIM $\mathrm{J}$ et al. Association of preoperative plasma levels of insulinlike growth factor I and insulin-like growth factor binding proteins- 2 and- 3 with prostate cancer invasion, progression, and metastasis. J Clin Oncol 2002; 20: 833-841. doi: 10.1200/ JCO.2002.20.3.833

[30] RINGER L, SIRAJUDDIN P, TRICOLI L, WAYE S, CHOUDHRY MU et al. The induction of the p53 tumor suppressor protein bridges the apoptotic and autophagic signaling pathways to regulate cell death in prostate cancer cells. Oncotarget 2014; 5: 10678-10691. doi: 10.18632/oncotarget. 2528

[31] OSMAN I, DROBNJAK M, FAZZARI M, FERRARA J, SCHER HI et al. Inactivation of the $\mathrm{p} 53$ pathway in prostate cancer: impact on tumor progression. Clin Cancer Res 1999; 5: 2082-2088.

[32] GROSSFELD GD, CARROLL PR, LINDEMAN N, MENG $\mathrm{M}$, GROSHEN S et al. Thrombospondin-1 expression in patients with pathologic stage T3 prostate cancer undergoing radical prostatectomy: association with $\mathrm{p} 53$ alterations, tumor angiogenesis, and tumor progression. Urology 2002; 59: 97-102.

[33] LAWLER J. Thrombospondin-1 as an endogenous inhibitor of angiogenesis and tumor growth. J Cell Mol Med 2002; 6: $1-12$.

[34] RAJAH R, VALENTINIS B, COHEN P. Insulin-like growth factor (IGF)-binding protein-3 induces apoptosis and mediates the effects of transforming growth factor-betal on programmed cell death through a p53- and IGF-independent mechanism. J Biol Chem 1997; 272: 12181-12188.

[35] SCHETTER AJ, HARRIS CC. Tumor suppressor p53 (TP53) at the crossroads of the exposome and the cancer genome. Proc Natl Acad Sci U S A 2012; 109: 7955-7956. doi: 10.1073/ pnas.1205457109

[36] NAUS CC, LAIRD DW. Implications and challenges of connexin connections to cancer. Nat Rev Cancer 2010; 10: 435441. doi: $10.1038 / \mathrm{nrc} 2841$

[37] SZPAK K, WYBIERALSKA E, NIEDZIALKOWSKA E, RAK M, BECHYNE I et al. DU-145 prostate carcinoma cells that selectively transmigrate narrow obstacles express elevated levels of Cx43. Cell Mol Biol Lett 2011; 16: 625-637. doi: 10.2478/s11658-011-0027-7

[38] CZYZ J, SZPAK K, MADEJA Z. The role of connexins in prostate cancer promotion and progression. Nat Rev Urol 2012; 9: 274-282. doi: 10.1038/nrurol.2012.14 